
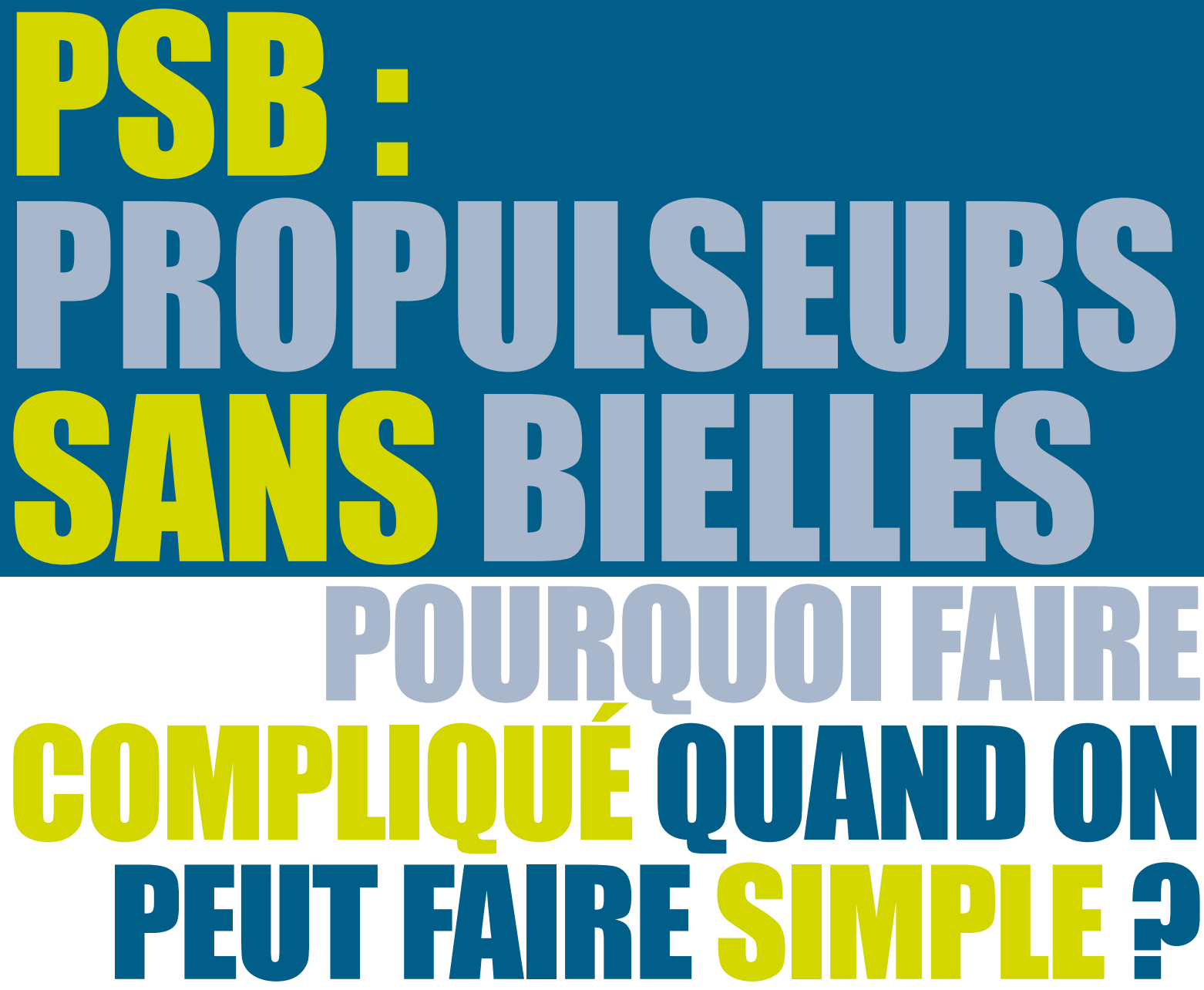

\title{
Antoine yArou
}


es classes II dento-squelettique sont associées à une absence de la fonction d'incision correcte du fait du surplomb incisif (absent dans les classe II, 2 et créé par la décompensation thérapeutique), au risque de fracture des incisives supérieures par leur position de parechoc et, sur le plan fonctionnel, à la déglutition primaire elle-même associée à une respiration mixte (nasale et buccale).

Le traitement orthopédique précoce est par conséquence l'indication de choix afin d'assurer la fonction d'incision des aliments, de limiter les risques de fracture des incisives supérieures et de permettre le plus tôt possible la rééducation de la déglutition, plus facile en l'absence d'un surplomb incisif.

Après avoir utilisé depuis des années des appareils fonctionnels (Frankel, Balters II) des gouttières avec des bielles de Herbst puis le PUL dans l'étape de la propulsion mandibulaire pour la correction des classe II, nous avons commencé par utiliser depuis moins d'un an des gouttières thermoformées dont la propulsion est assurée simplement par des élastiques intermaxillaires. L'idée est venue du Dr Philippe Frappier, exerçant l'ODMF à Bordeaux.

Avant d'aborder les détails de ces gouttières, nous présentons ci-dessous un aperçu des étapes du traitement orthopédique de la classe II que nous utilisons :

1- Expansion maxillaire : Si le diagnostic d'endomaxillie est posé (les dents supérieures latérales ne recouvrent pas les dents inférieures en classe I sur les moulages, le plancher nasal réel est plus petit que le plancher nasal théorique sur l'analyse de face de Delaire - TRIDIM), un quad'hélix est posé avant 7 ans (fermeture de la suture palatine médiane) et un disjoncteur après.

2-Expansion de l'arcade inférieure, en présence d'encombrement dentaire.

3-Nivellement de l'arcade supérieure : Au même titre que ce qui se fait en préparation orthodontique avant ostéotomie mandibulaire sagittale d'avancée dans les classe II chez l'adulte, un nivellement au moins de l'arcade supérieure en denture mixte est réalisé, il a pour but d'assurer le maximum de stabilité occlusale après propulsion mandibulaire et de prévenir une inclusion des canines supérieures, surtout si la classe II est associée à un encombrement de l'arcade supérieure. Le patient est donc appareillé deux fois en multiattâches : une fois en denture mixte et une dernière fois en denture définitive.

4-Propulsion mandibulaire : l'appareillage fixe est déposé le même jour le matin avec prise d'empreinte puis deux gouttières thermoformées sont posées en fin de journée afin de ne pas perdre le bénéfice des résultats obtenus lors des étapes précédentes.

Nous avons été agréablement surpris de constater que la correction de la classe II se fait la plupart des fois plus rapidement qu'avec les autres propulseurs à système de bielles qui sont abandonnés depuis, et ceci même avec un port seulement nocturne.

\section{BONFECTION DES GOUTTIËRES}

Nous avons commencé à réaliser nos premières gouttières en utilisant l'ESSIX machine de Sheridan et les plaques associées. Cet appareil procède par aspiration.

Pour des raisons de précision et de stabilité, cette pratique a été abandonnée aujourd'hui au profit des plaques Duran utilisées avec le Biostar de Scheu Dental, dont le principe est basé sur la compression.

La gouttière est de $2 \mathrm{~mm}$ d'épaisseur,qui diminue après le thermoformage. Les plaques de Duran sont plus précises que les plaques A+ de Schéridan, à condition de supprimer la feuille qui sert $d$ 'isolant (Isofolan). Pour la fixation des élastiques intermaxillaires de classe II, un crochet, fabriqué à l'aide de résine (Orthorésine), est collé sur le collet de la molaire inférieure et un autre sur la canine. Afin d'éviter la bascule du plan d'occlusion lors des tractions élastiques, des forces de faible amplitude sont utilisées. 

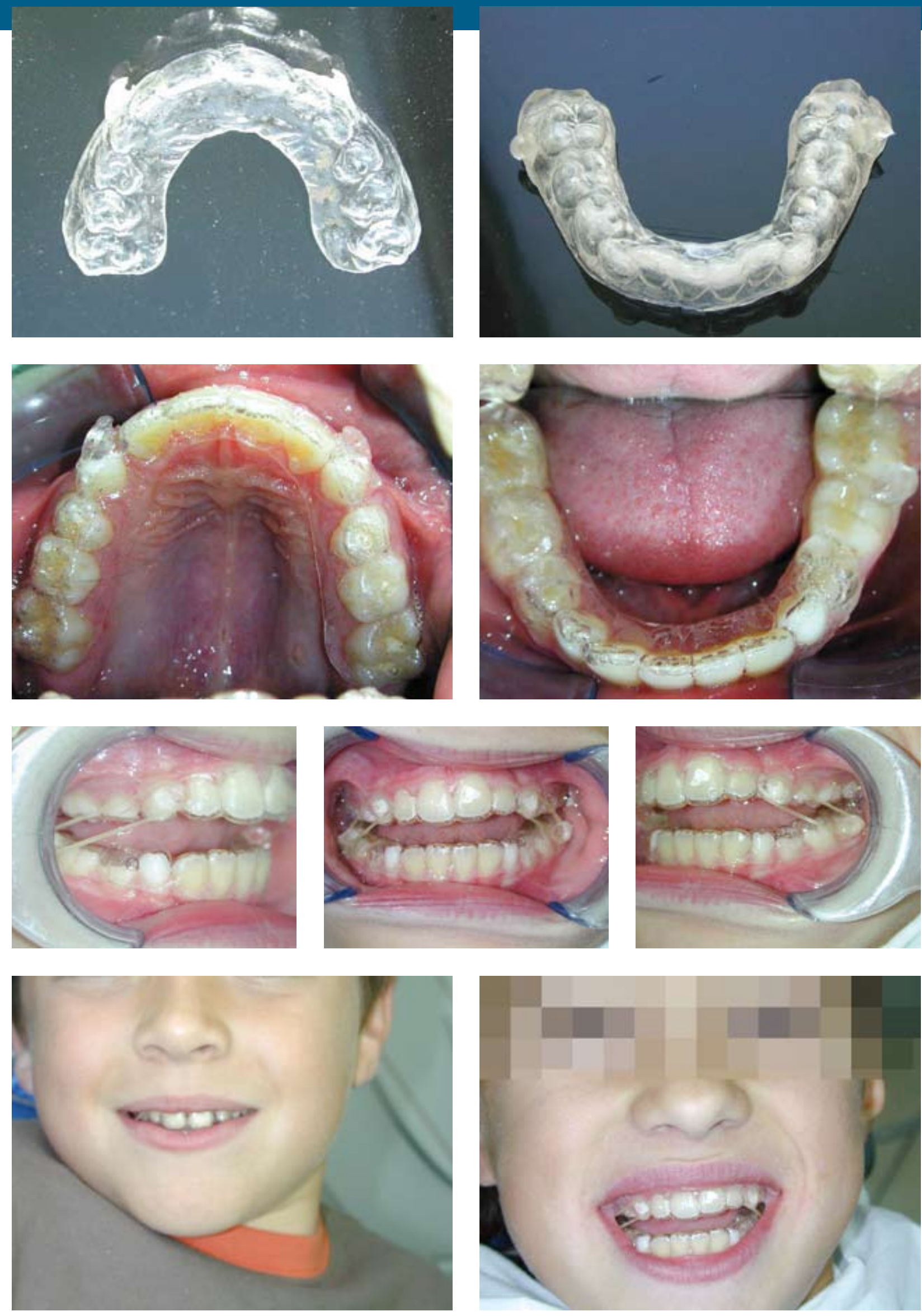


\section{Bis $K^{\circ} 2$}

\section{SYNTHESE}

Les chiffres donnés dans ce tableau correspondent aux differences ontro hos chiffres optimaux of coux du pationt

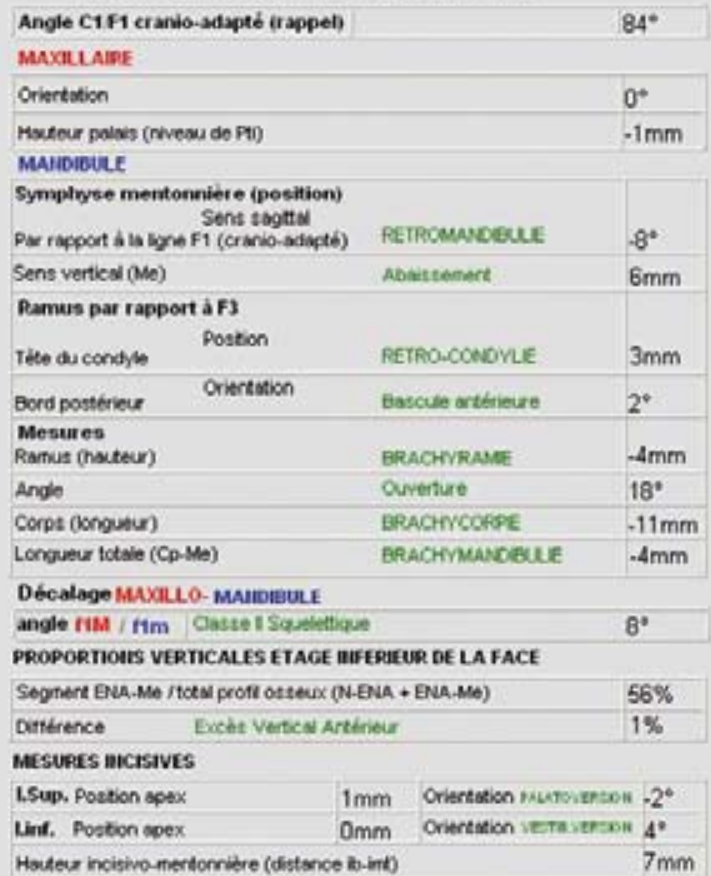

Hatecs incisivomentomitre (distance to int)

\section{OS HYOHOE}

Nveou Hy por ropport \& V3 aboissemert $\quad \nabla 3 \mathrm{~mm} \quad \Delta$
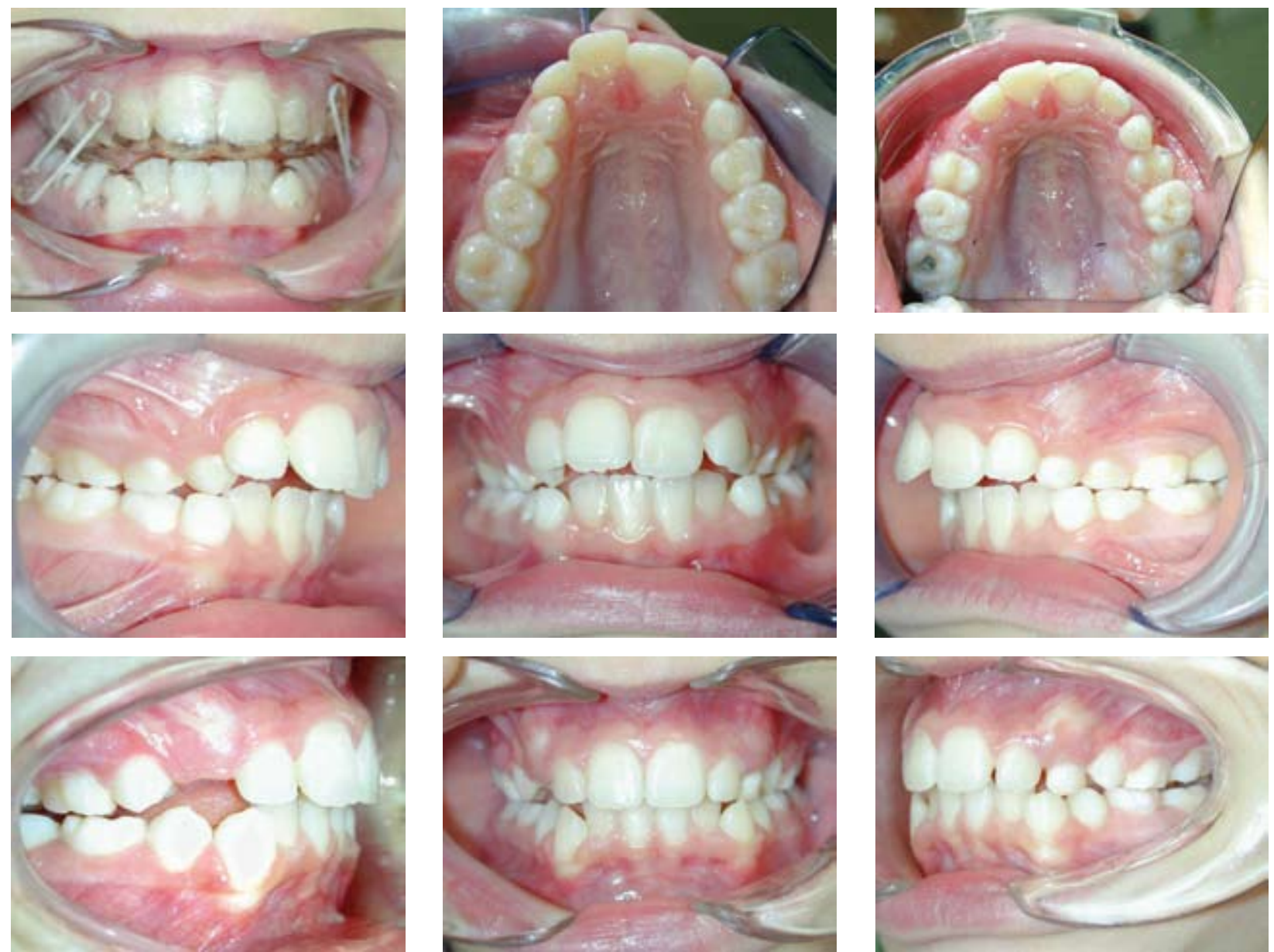

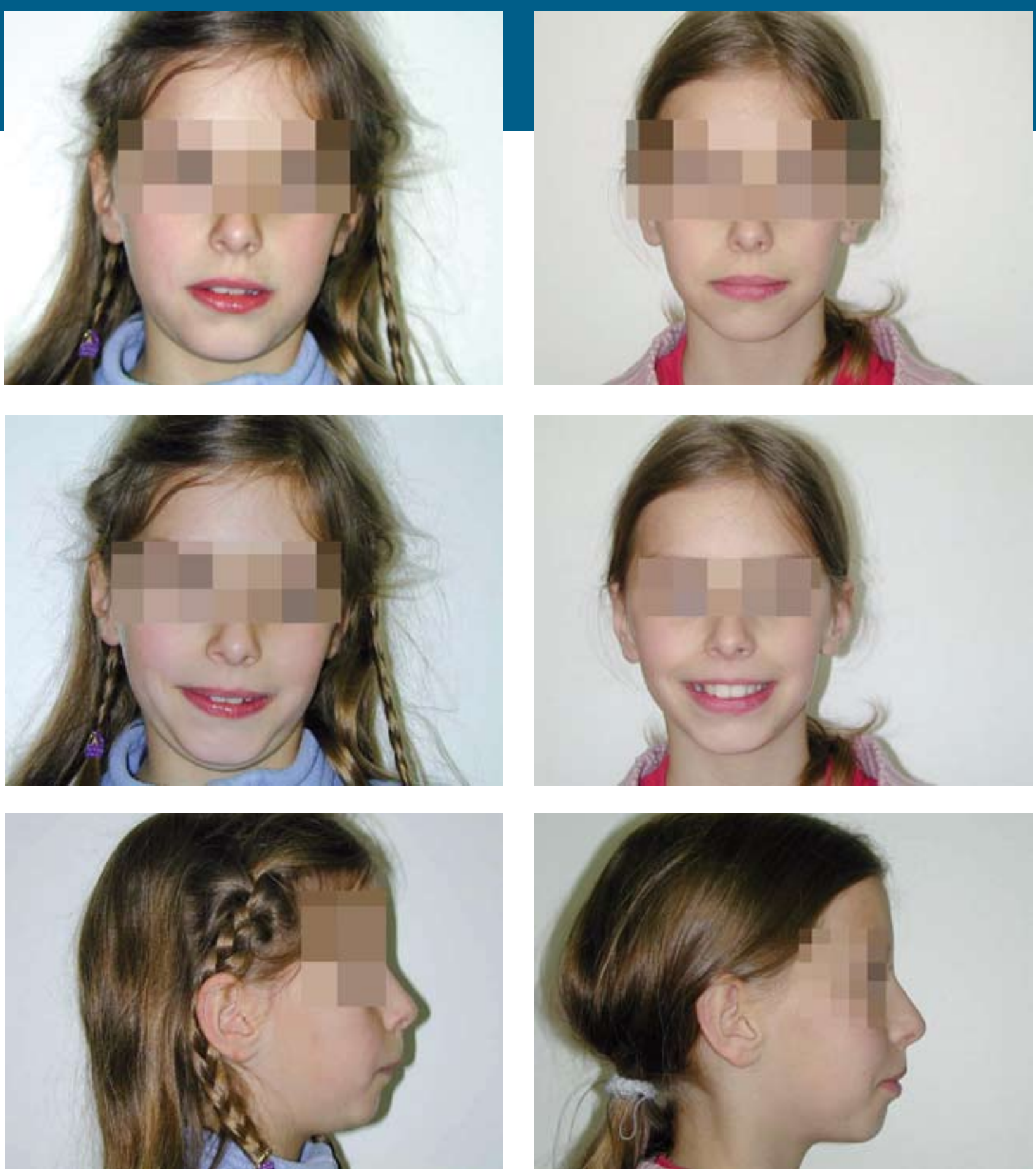

Patiente présentant une classe II dentosquelettique par rétromandibulie, associée à un excès vertical antérieur de l'étage inférieur facial et une insuffisance verticale postérieure.

\section{PLAN THÉRAPEUTIQUE}

Expansion maxillaire et nivellement de l'arcade supérieure : 8 mois

Gouttière supérieure avec plan de surélévation rétro-incisif : 3 mois

Pose de système GSB : obtention d'une presque classe I dentaire mais instable 3 mois plus tard, à

9 ans et 9 mois et prise de photos. Quelques mois de port seront encore probablement nécessaires pour consolider la classe I dentaire. À noter que nous n'avons pas attendu le pic de croissance pour intercepter la classe II hyperdivergente.

La rééducation de la déglutition secondaire associée, assurée par l'équipe du cabinet, peut déjà commencer en attendant la denture définitive, date de reprise en fixe pour la finition. Une génioplastie fonctionnelle d'ascension et d'avancée du menton pour corriger la dysfonction labiomentonnière et établir la classe I squelettique sera éventuellement à évaluer en fin de croissance. 
À noter l'amélioration du profil par la réduction du bombé de la lèvre supérieure, par l'avancée de la lèvre inférieure et du menton, la diminution de la crispation labio-mentonnière et enfin l'amélioration du rapport des incisives supérieures avec la lèvre inférieure.

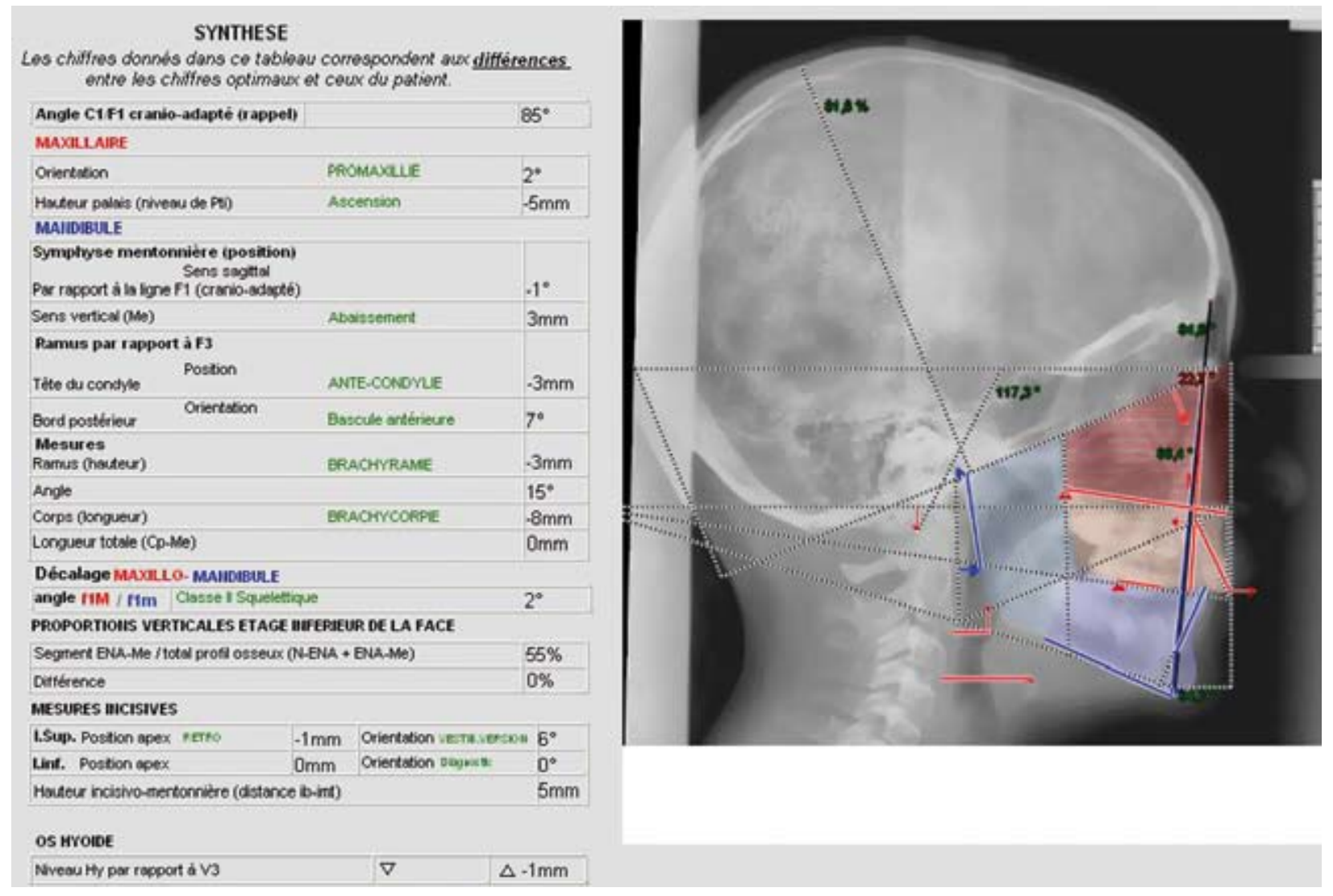

Cas de classe II avec dysharmonie dentomaxillaire. La patiente a bénéficié d'une expansion maxillaire, d'un nivellement de l'arcade inférieure et d'une ingression des incisives inférieures à l'aide d'un plan palatin rétroincisif supérieur sur gouttière avant la propulsion mandibulaire de manière à assurer l'occlusion la plus stable lors de la propulsion.

Un système de PSB a été posé par la suite le même jour de la dépose. On observe un début de correction vers la classe I, quatre mois après le port. À cette date, les gouttières, surtout l'inférieure, commencent à devenir instables du fait de l'éruption des canines et des premières prémolaires inférieures. Deux possibilités s'offrent alors : fraiser la résine en regard de ces dents pour libérer de la place ou fabriquer de nouvelles gouttières. La deuxième option est retenue.

\section{WNTERETS DU GSB}

Les tarifs des bielles et du PUL ne cessant d'augmenter ainsi que celui des charges des cabinets, et celui du remboursement des caisses n'évoluant pas, il a fallu chercher une alternative efficace dont la fabrication ne nécessite pas au moins deux heures, comme celle des Bielles de Herbst ou du PUL, et disponible le jour même de la dépose, du fait de l'absence totale d'un squelette métallique dans ses composants.

La fabrication simple du GSB est possible au cabinet dans un délai bref surtout dans le cas d'utilisation d'un plâtre rapide.

L'absence de métal minimise les risques d'allergies (ex : nickel)

Lors de la propulsion mandibulaire avec les autres appareils (Bielles de Herbst, PUL) 

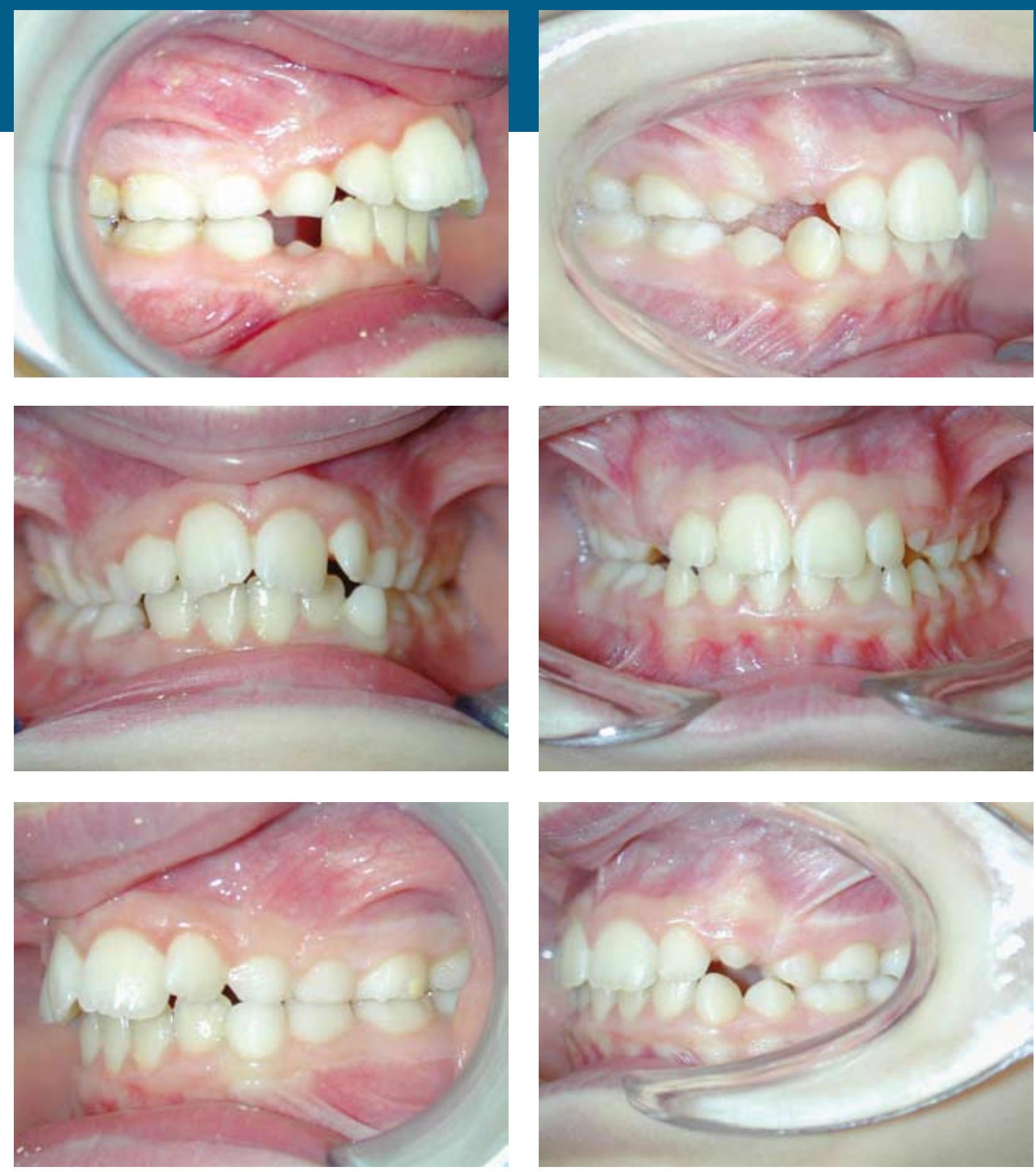

on observe une instabilité due à l'éruption principalement des canines et des premières prémolaires. Pour y remédier, il est nécessaire de supprimer une partie du squelette métallique à section rectangulaire des bielles (ce qui est impossible sans refaire tout l'appareil) ou du PUL (ce qui le fragilise compte tenu qu'il est évidé le long de son axe principal). Cette problèmatique n'existe pas dans le GSB puisqu'il est possible de fraiser la résine au niveau de la dent concernée ou refaire une autre gouttière en un laps de temps très court si le problème concerne plusieurs dents.

son port dans la journée.

Il est possible de commencer la traction élastique dès le début du nivellement de l'arcade supérieure en denture mixte, en combinant une gouttière inférieure. Ceci se fait en accrochant l'élastique sur un bracket de la canine supérieure et sur le crochet de la gouttière inférieure, ce qui permettra d'optimiser la propulsion mandibulaire.

Nous espérons que cet article suscitera les avis et les commentaires des différents utilisateurs afin d'améliorer toujours nos résultats orthodontiques. Son aspect transparent, donc esthétique, permet 